\title{
Supplementary Information Sheet
}

\section{Green Synthesis of Graphene Based Biomaterial using Fenugreek Seeds for Lipid Detection}

\author{
Chandan Singh, Md. Azahar Ali, Gajjala Sumana*
}

Figure S1

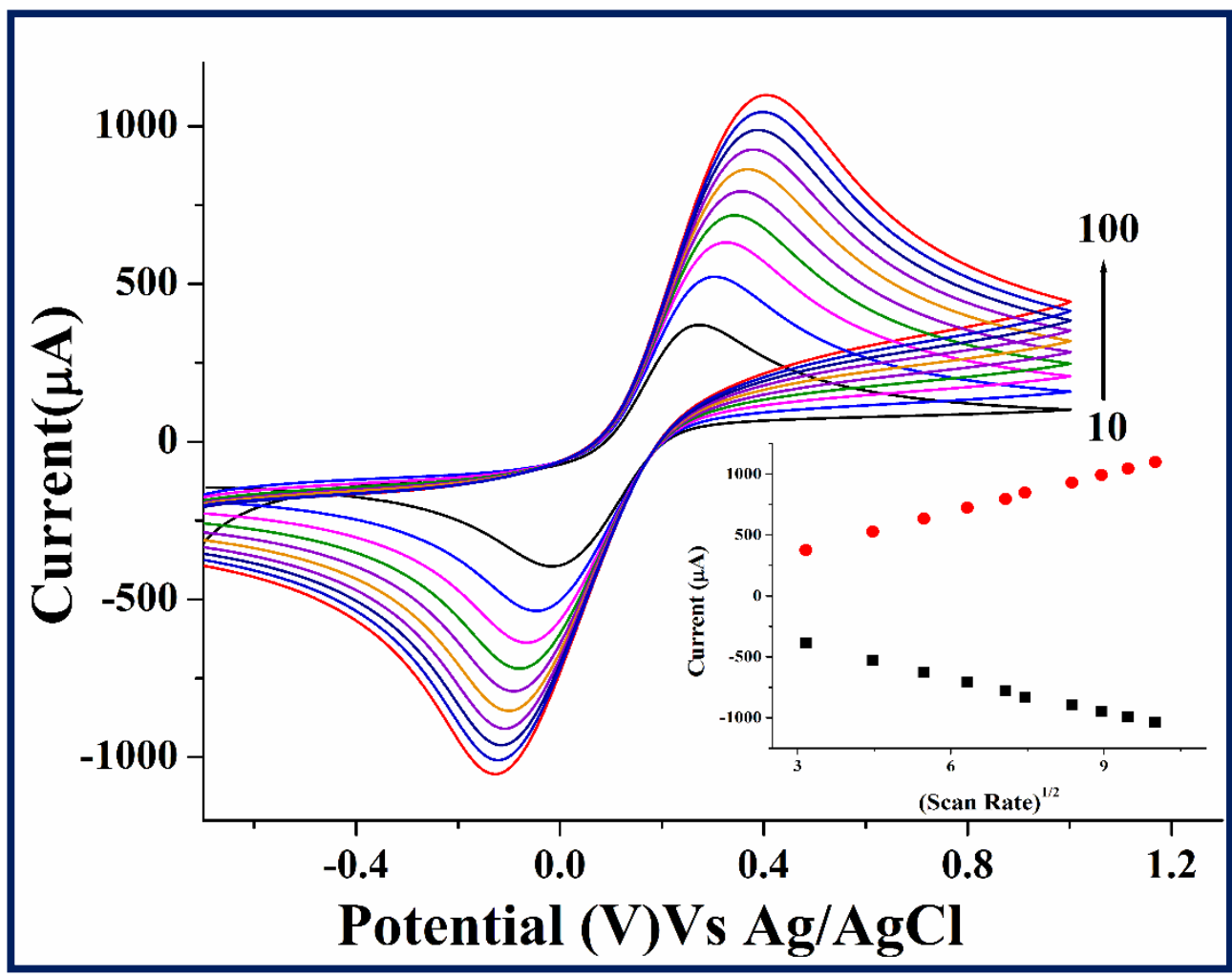

Figure S1: CV analysis of LIP-GLDH/Pd-rGO electrode as a function of scan rate [10-100 $\mathrm{mV} / \mathrm{s}]$ and anodic/cathode peak current with respect to square root of scan rate (Inset). 
Figure S2

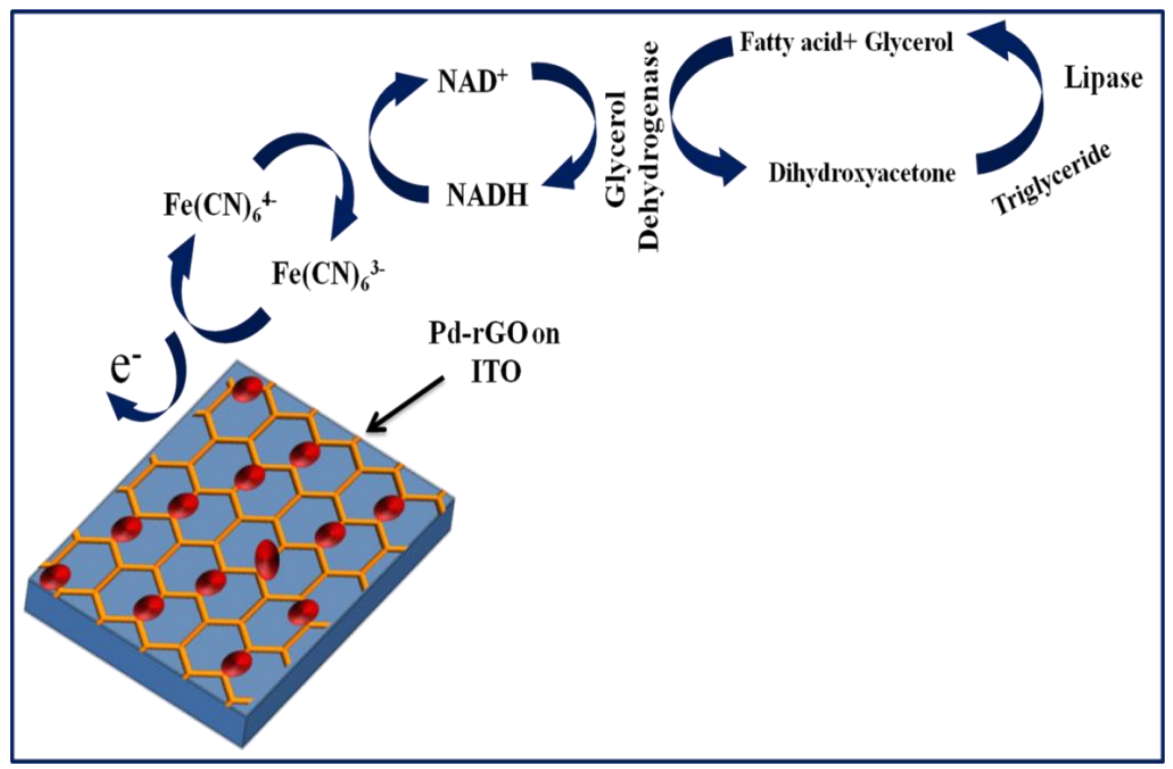

Figure S2: Electrochemical reactions pathway and generation of electron due to the reaction. 
Figure S3

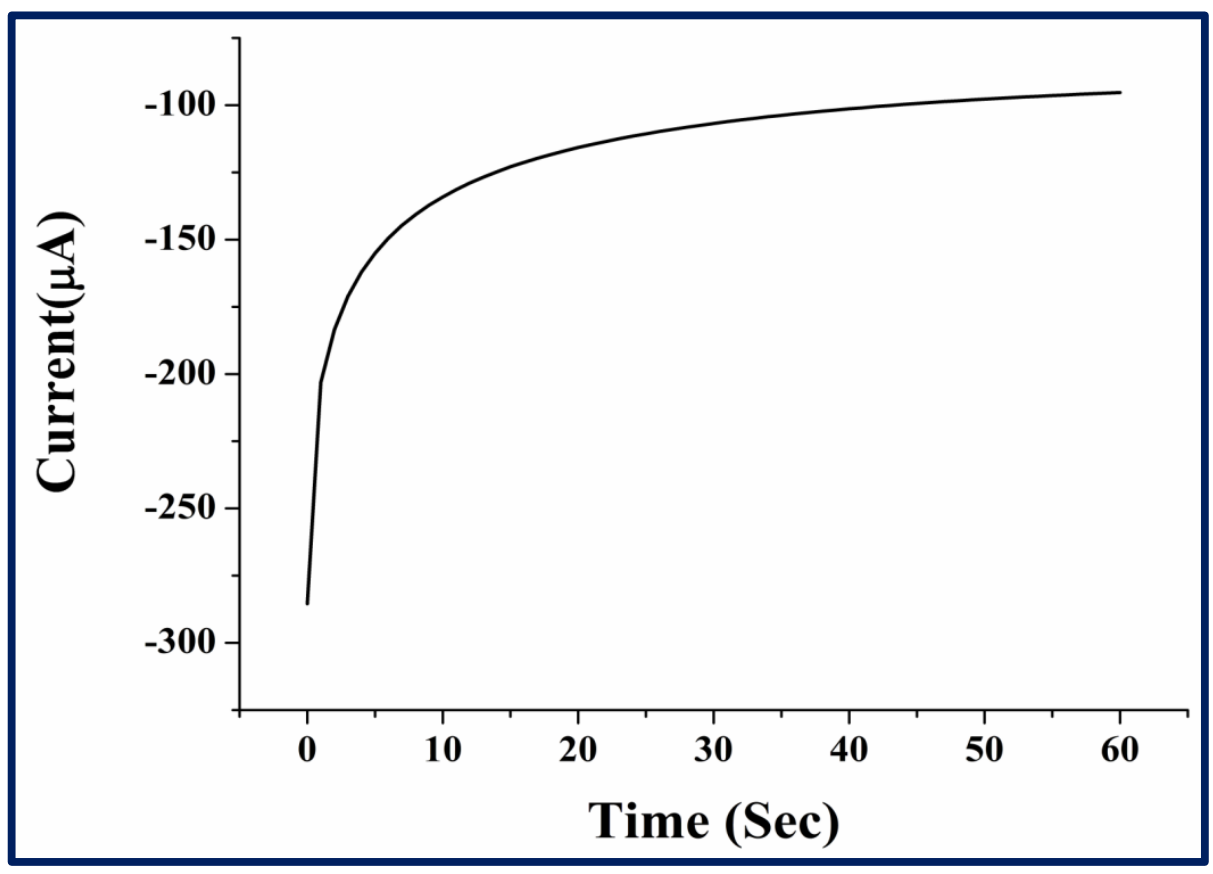

Figure S3: Hanes-Woolf plot for the biosensing response of LIP-GLDH/Pd-rGO.

Figure S4

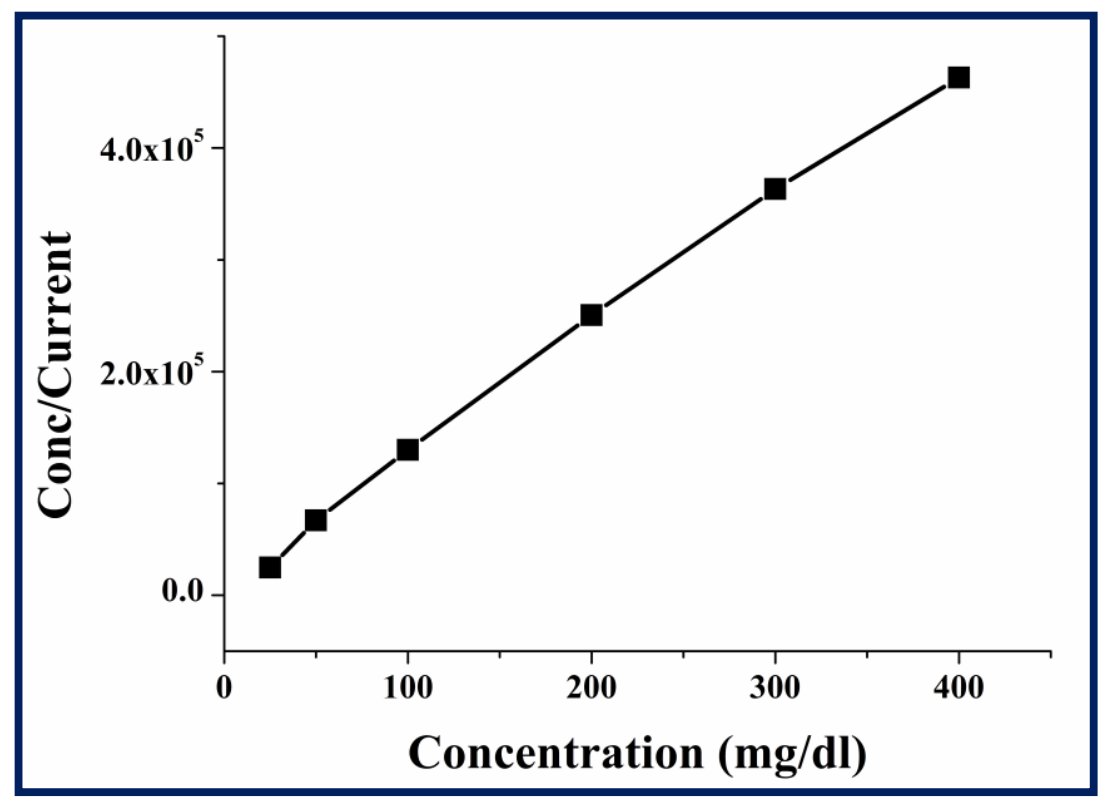

Figure S4: Chronoamperometric responses of LIP-GLDH/Pd-rGO. 
Figure S5

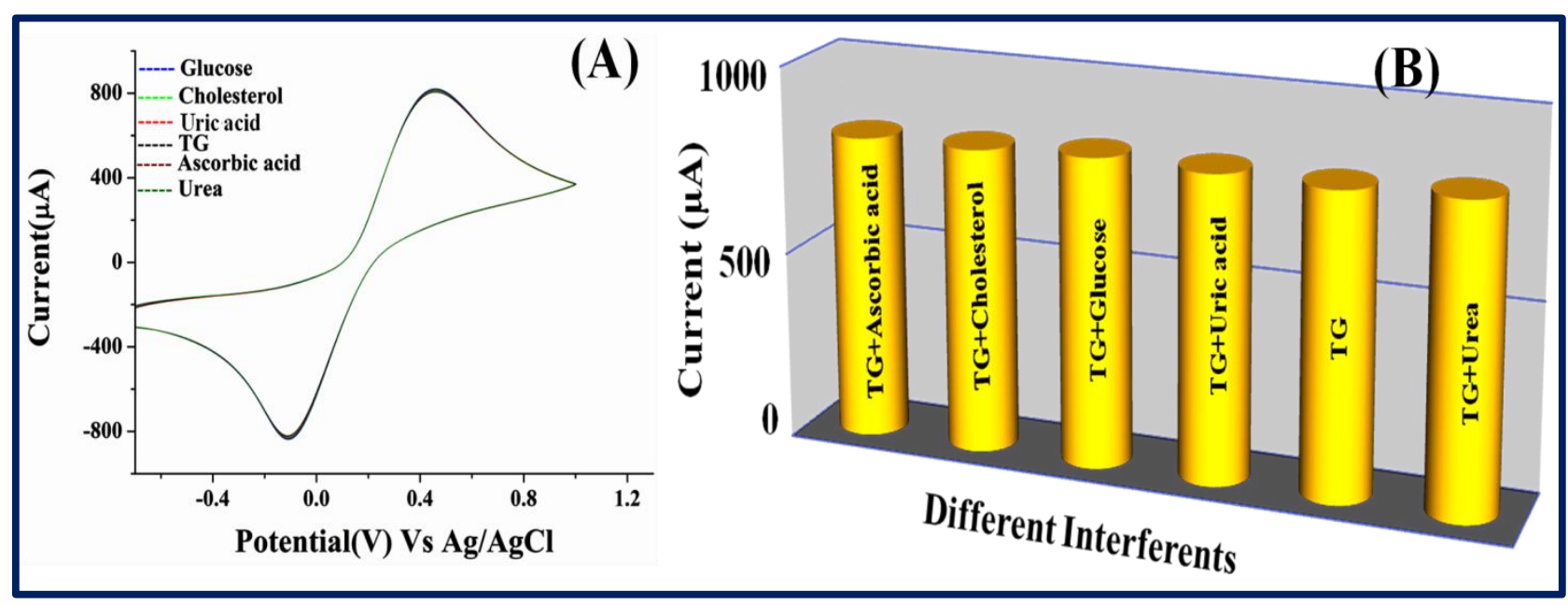

Figure S5: Interference study of LIP-GLDH/Pd-rGO electrode in presence of various possible interferents.

Figure S6

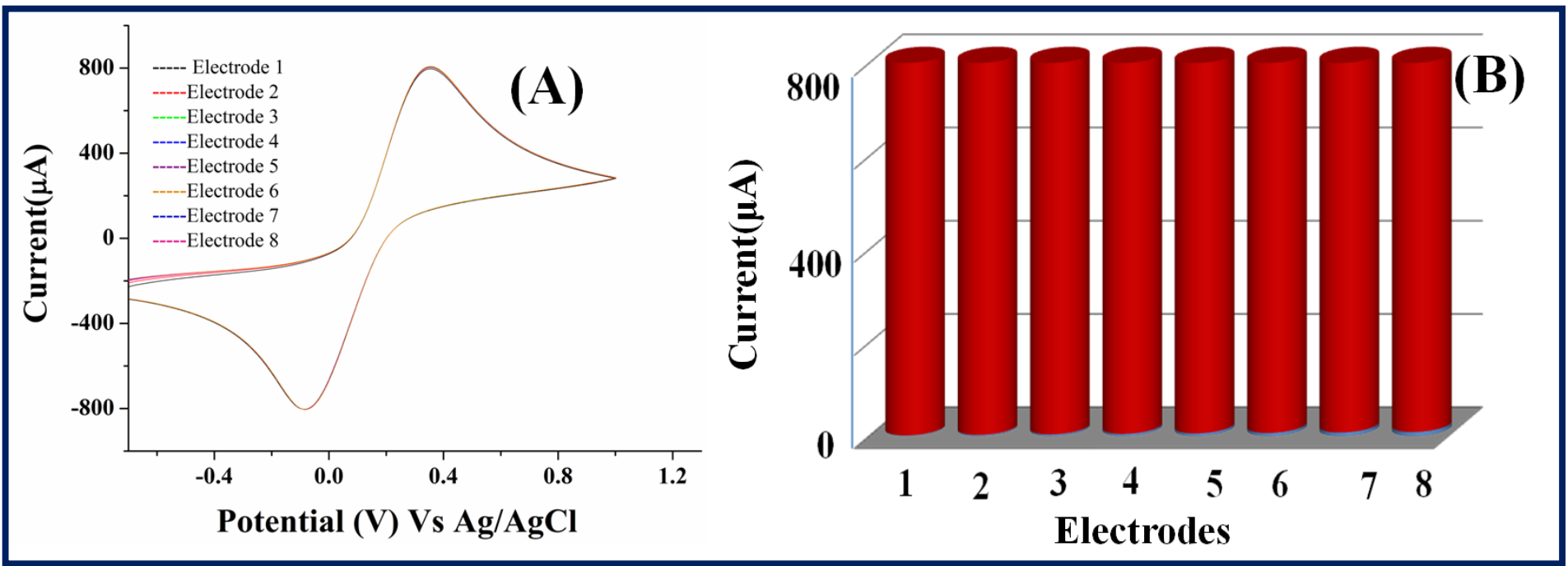

Figure S6： Repeatability studies of LIP-GLDH/Pd-rGO electrode. 
Figure S7

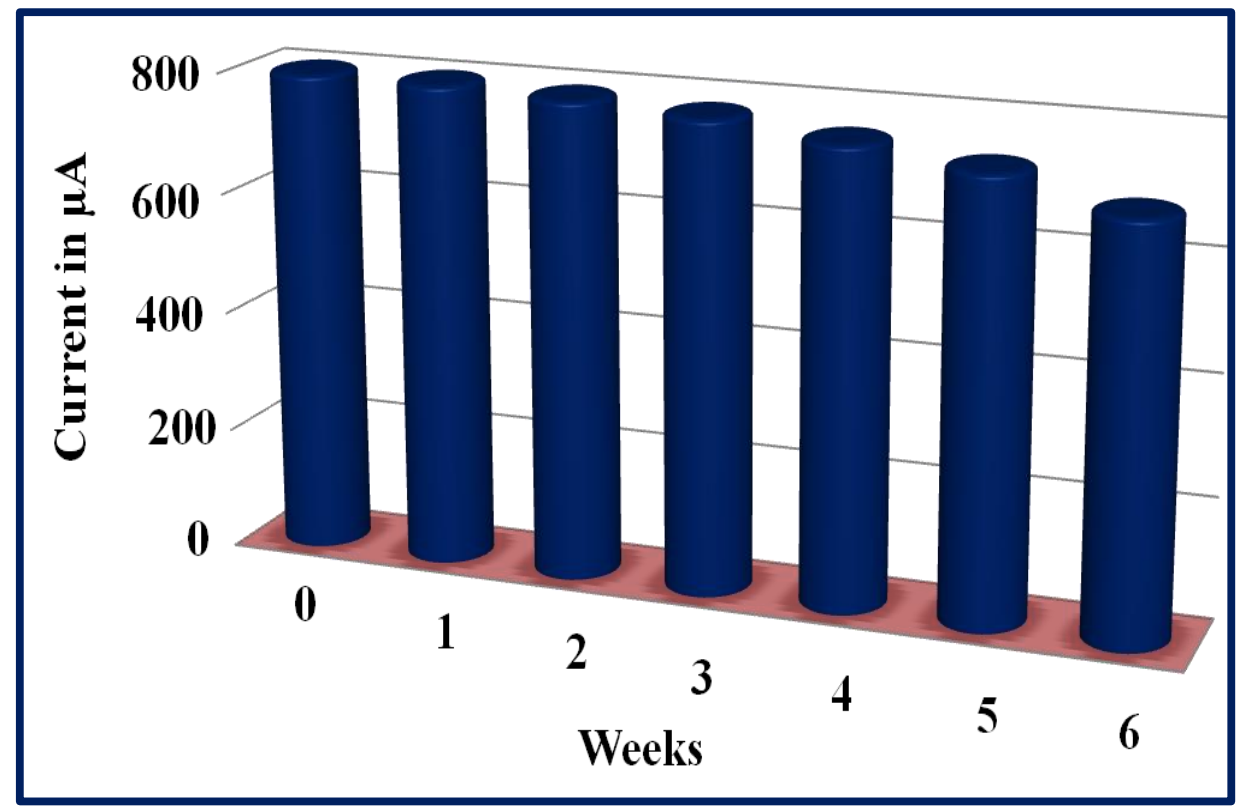

Figure S7 : Stability studies of LIP-GLDH/Pd-rGO electrode. 